\title{
An Optimized Design of Single-Channel Beta-Gamma Coincidence Phoswich Detector by Geant4 Monte Carlo Simulations
}

\author{
Weihua Zhang, Pawel Mekarski, Marc Bean, Jing Yi, and Kurt Ungar \\ Radiation Protection Bureau of Health Canada, 775 Brookfield Road, AL 6302D1, Ottawa, ON, Canada K1A 1C1 \\ Correspondence should be addressed to Weihua Zhang, weihua.zhang@hc-sc.gc.ca
}

Received 8 November 2010; Revised 31 January 2011; Accepted 16 March 2011

Academic Editor: Luigi Petrizzi

Copyright (c) 2011 Weihua Zhang et al. This is an open access article distributed under the Creative Commons Attribution License, which permits unrestricted use, distribution, and reproduction in any medium, provided the original work is properly cited.

\begin{abstract}
An optimized single-channel phoswich well detector design has been proposed and assessed in order to improve beta-gamma coincidence measurement sensitivity of xenon radioisotopes. This newly designed phoswich well detector consists of a plastic beta counting cell (BC404) embedded in a $\mathrm{CsI}(\mathrm{Tl})$ crystal coupled to a photomultiplier tube. The BC404 is configured in a cylindrical pipe shape to minimise light collection deterioration. The CsI(Tl) crystal consists of a rectangular part and a semicylindrical scintillation part as a light reflector to increase light gathering. Compared with a PhosWatch detector, the final optimized detector geometry showed $15 \%$ improvement in the energy resolution of a ${ }^{131 \mathrm{~m}} \mathrm{Xe} 129.4 \mathrm{keV}$ conversion electron peak. The predicted betagamma coincidence efficiencies of xenon radioisotopes have also been improved accordingly.
\end{abstract}

\section{Introduction}

In the Comprehensive Nuclear Test Ban Treaty (CTBT) verification regime, the two-dimensional beta-gamma coincidence detector is widely used for radioxenon activity measurement due to its ability to distinguish ${ }^{131 \mathrm{~m}} \mathrm{Xe}$ and ${ }^{133 \mathrm{~m}} \mathrm{Xe}$ and to suppress noncoincident background events as well as for its high sensitivity to the coincident events characteristic of the xenon radioisotopes of interest [1]. The beta-gamma coincidence detector basically consists of two scintillators, that is, an inorganic scintillator and a plastic scintillator counting cell. In many beta-gamma coincidence systems, beta and gamma radiations are detected in two separate channels, such as the automated radioxenon sampler/analyzer [2], the Swedish unattended noble gas analyzer [3], and the automatic portable radiometer of isotopes xenon [4]. To simplify the coincidence system configuration and calibration procedure, a phoswich, namely PhosWatch (PW5), beta-gamma coincidence detector, has been developed, in which both beta and gamma radiations can be detected by one single channel [5]. The PW5 detector has a cavity drilled inside the inorganic scintillator, which accommodates the plastic scintillator counting cell
(BC404). The embedded plastic cell is filled with xenon gas to be counted. The xenon radioisotope decays by emitting gamma-rays or X-rays in coincidence with beta-particles or conversion electron $(\mathrm{CE})$, respectively. The plastic scintillator is used to absorb all beta-particles and CE, while the longer range gamma-rays and $\mathrm{X}$-rays are mainly detected by inorganic scintillator. When a xenon radioisotope emits both $\mathrm{CE}$ and $\mathrm{X}$-rays, beta-gamma coincident signal can be distinguished. The detector enables the same energy $(30.0-\mathrm{keV})$ $\mathrm{X}$-rays emitted from ${ }^{131 \mathrm{~m}} \mathrm{Xe}$ (abundance $44.2 \%$ ) and ${ }^{133 \mathrm{~m}} \mathrm{Xe}$ (abundance $45.7 \%$ ) to be separated by the well-specified discrete conversion electron peaks at $129.4-\mathrm{keV}$ (abundance $60.7 \%$ ) and $198.7-\mathrm{keV}$ (abundance 63.1\%), respectively. The peak width of CE and X-rays is mostly limited by the resolution of the scintillators, but the spatial variations in the light collection efficiency throughout the detector can significantly degrade the overall energy resolution of the detector.

It was shown previously that the degraded X-ray and CE peak energy resolutions due to light collection efficiency deterioration (light-path obstruction) by the embedded beta counting cell can be minimised by varying the embedded beta counting cell positions inside the CsI(Tl) crystal [6]. The 
previous simulation demonstrated that the PW5 detector configuration obtained by placing beta counting cell furthest away from the photomultiplier tube (PMT) can provide the best overall CE energy peak resolution [6]. However, in this configuration, some gamma-rays may escape out of the thinner part of $\mathrm{CsI}(\mathrm{Tl})$ crystal, which may result in a lower beta-gamma coincidence measurement sensitivity of xenon radioisotopes, especially for ${ }^{135} \mathrm{Xe}$ due to its relatively high energy gamma-ray $(249.8 \mathrm{keV})$. For this reason, it is worthwhile to investigate an alternative phoswich detector design that will have both optimal CE and X-ray energy resolution and beta-gamma coincidence efficiency for all xenon radioisotopes. The design guidance, incorporates the following key principles: single-channel phoswich detector for keeping system simplicity minimization of lost or obstructed photons by embedding the beta counting cell between the inorganic scintillator and the PMT availability of a commercial scintillator and ease of manufacturing and the shape of $\mathrm{CsI}(\mathrm{Tl})$ scintillator for maximum light gathering by optical reflection.

\section{Monte Carlo (MC) Simulation for Detector Geometry Design}

An experimentally determined pure ${ }^{131 \mathrm{~m}} \mathrm{Xe}$ standard betagamma coincidence spectrum by PW5 detector is illustrated in Figure 1. The two dimensional histograms (c) and (d) at the bottom are distributions resulting from energy deposition events in the BC404 (beta-energy axis) and CsI(Tl) (gamma-energy axis) detectors, respectively. The top view of three dimensional histogram at the top right (b) contains information regarding the beta-gamma coincidence decay events detected by the PhosWatch detector. The histogram is divided into several rectangularly shaped regions of interest (ROI) based on the gamma-ray energy and beta-particle energy calibrations. The boundaries of each ROI rectangular area are in energy units and depend on xenon radioisotope decay modes. The gross counts in each ROI are calculated by summing the counts per channel and used to calculate the radioxenon concentration, as listed in Figure 1(a).

As shown in Figure 1(d), the energy resolution of the prototype PW5 (beta counting cell located at geometry centre of CsI(Tl) detector) at ${ }^{131 \mathrm{~m}} \mathrm{Xe} 129.4 \mathrm{keV}$ CE peak is experimentally determined by a pure ${ }^{131 \mathrm{~m}}$ Xe source as $38.0 \%$, which is expressed as peak full width at half maximum (FWHM) divided by peak center position. Subsequently, the optical resolution scale of BC404 scintilltor was defined such that the simulated FWHM at $129.4 \mathrm{keV}$ CE peak matches this experiment's value. The resolution scale was also applied to the following steps in the new phoswich detector geometry design.

There are several steps involved in performing the proposed phoswich detector design and optimization. The first step of simulation design involved the addition of an identical PMT to the face opposite the original PMT window. This additional PMT collected the light directed to the other half of the scintillator crystal as opposed to reflecting it back around the inner scintillator shell. The second step involved altering the geometry of the PW5 detector itself in order to reflect the maximum amount of light possible into the PMT. This involves finding the optimum of CE and X-ray energy resolution and beta-gamma coincidence efficiency by varying the embedded plastic cell positions inside the $\mathrm{CsI}(\mathrm{Tl})$ detector, and the analysis of several different types of reflectors on the surface opposite the PMT window.

All the above detector geometry and designs evaluations were performed with $\mathrm{MC}$ simulations according to the ${ }^{131 \mathrm{~m}} \mathrm{Xe} \mathrm{CE}$ energy peak resolution. The MC simulations were created using the GEANT4 Toolkit [7]. All of the data processing and output were performed through the implementation of AIDA [8] in the simulation. GEANT4 has also been shown to accurately simulate the PW5 detector system, including the optical signals received by the PMT [9]. The identical simulation methodology has been applied for this study with any changes to geometry or tracking explained. GEANT4 allows the user to define the geometry and physics processes to be used in the simulation. In this simulation, both the standard electromagnetic and optical process packages were defined. GEANT4 then handles the transportation and interaction of the simulated particle through the defined geometry. The radioactive decay module (G4RadioactiveDecay.3.2) was used for radioxenon isotopes. For each radioxenon decay event, the Geant4 detector module records the energy deposition in each scintillator and counts the number of photons captured by the PMT from each scintillator, with a time stamp. The time difference between the BC-404 plastic cell and the $\mathrm{CsI}(\mathrm{Tl})$ scintillator is recorded, and events occurring within a specified "coincidence-time window" of each other in the respective scintillator are considered to be coincident. At the end of the event, the total photons captured by the PMT from each scintillator as photon pairs are stored in a data table within an AIDA object as raw data to produce different histograms.

\section{Results and Discussion}

The simulation results demonstrated that through the modification of the original PW5 detector geometry, a noticeable improvement in FWHM was achieved. With the BC404 beta counting cell placed in the centre of the $\mathrm{CsI}(\mathrm{Tl})$ coupled with dual PMT on the opposite side of CsI(Tl) crystal, the FWHM obtained was $32.7 \%$. Compared with the original single PMT design, a $16 \%$ improvement was achieved. Due to the fact that almost all photons that travel to either side of the crystal are detected by the respective PMTs, without needing to be reflected back around, this FWHM given by dual PMT phoswich detector design was taken as the maximum theoretical value, and was used as the limit that can be achieved by a single-channel phoswich detector configuration. The resulting single-channel phoswich detector design was then optimized by changing the detector geometry in order to create better light collection and ultimately to approach the maximum theoretical FWHM value.

The first design change was the position of the BC404 beta counting cell within the cylindrical CsI(Tl) scintillator [6]. The position was varied along the axis perpendicular to the face of the PMT. The FWHM of ${ }^{131 \mathrm{~m}}$ Xe CE peak at 


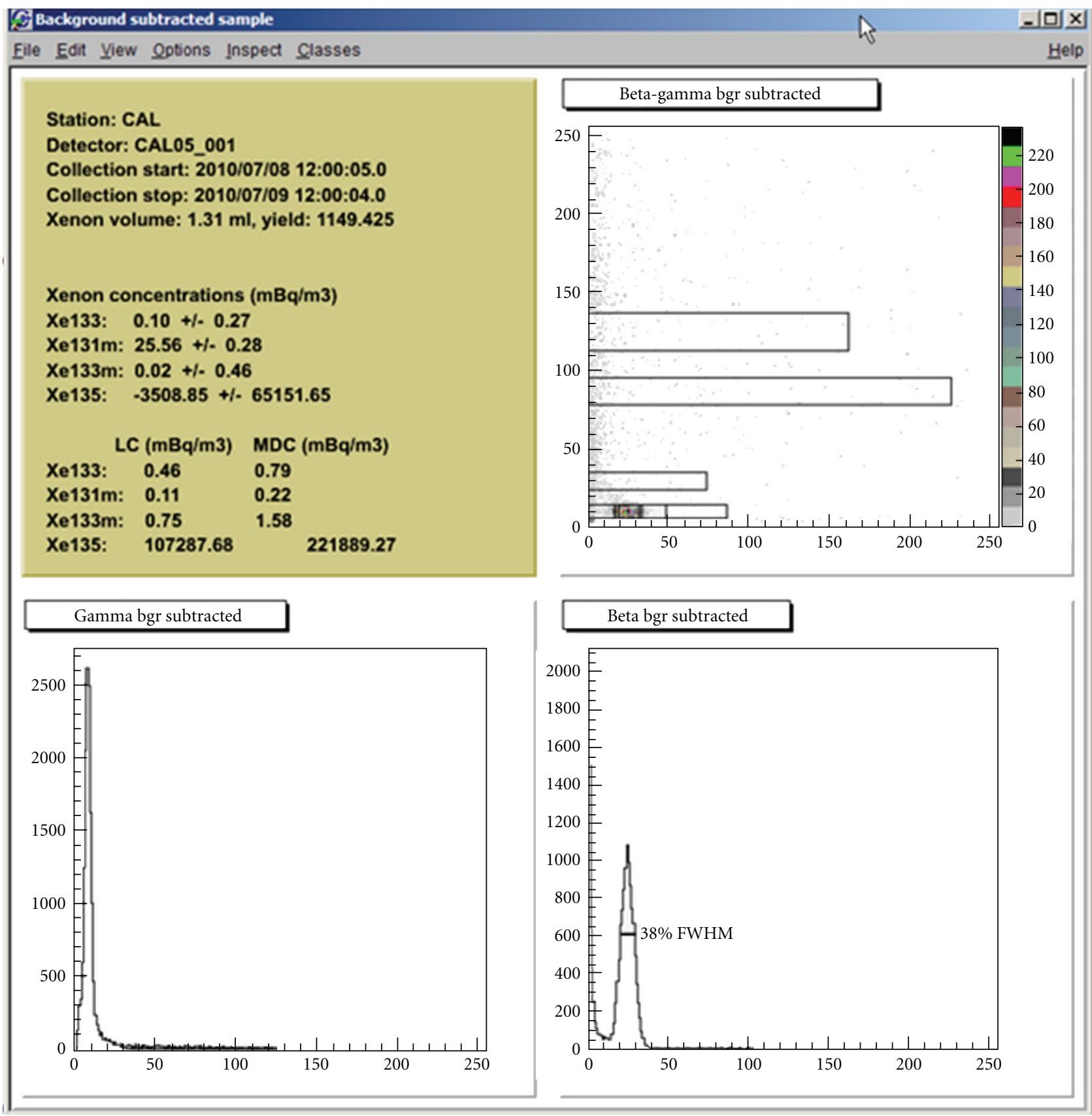

FIgURE 1: An experimentally determined pure ${ }^{131 \mathrm{~m} X e}$ standard beta-gamma coincidence spectrum by PW5 detector. The analyzed radioxenon concentration (a). Top view of the 3D histogram regarding the beta-gamma coincidence decay events detected by PhosWatch detector (b). The 2D histograms of energy deposition events at $\mathrm{CsI}(\mathrm{Tl})$ (c) and BC404 (d).

these beta counting cell positions was analysed by varying the cell-PMT distance with the original centre position as reference point. The results show that there is actually a decrease in FWHM for a position close to the PMT face. However, for positions farther away from the PMT, there is an improvement in the FWHM of the system. The simulation demonstrated that the detector configuration obtained by placing the beta counting cell furthest away from the PMT provides the best detector energy resolution. A 9\% improvement in the FWHM of the $129.4 \mathrm{keV} \mathrm{CE}$ of ${ }^{131 \mathrm{~m} X e}$ was achieved compared to the FWHM value obtained with the beta counting cell at the $\mathrm{CsI}(\mathrm{Tl})$ geometric centre [6].

The second modification was to further minimise the peak broadening due to light path interruption caused by the embedded beta counting cell. The redesign proposed was to change the previous flat $\mathrm{CsI}(\mathrm{Tl})$ surface shape (opposite side of the PMT), to a cylindrical shaped reflector. The idea was that the reflectors should help to reflect photons from the beta counting cell. In theory, this would reflect light originating from the counting cell perpendicular to the 
TABLE 1: The coincidence detection efficiencies $\left(\varepsilon_{\beta \gamma}\right)$ of the different X-rays and gamma rays of xenon radioisotopes by Geant4 modeling.

\begin{tabular}{|c|c|c|c|c|}
\hline \multirow[t]{2}{*}{ Radioxenon } & \multicolumn{2}{|c|}{ ROI limits, keV } & \multirow[b]{2}{*}{ ROI index } & \multirow[t]{2}{*}{$\varepsilon_{\beta \gamma}, \%$} \\
\hline & $y$-axis $(\mathrm{CsI})$ & $x$-axis (BC404) & & \\
\hline \multirow[t]{6}{*}{${ }^{133} \mathrm{Xe}$} & $17.47-42.59$ & $0-347.50$ & 1 & $89.6(87.2)$ \\
\hline & $67.80-98.73$ & $0-396.50$ & 2 & $72.5(68.5)$ \\
\hline & $17.47-42.59$ & $16.25-89.55$ & 3 & $33.0(32.6)$ \\
\hline & $17.47-42.59$ & $239.03-396.504$ & 4 & $8.8(8.0)$ \\
\hline & $17.47-42.59$ & $170.07-396.505$ & 5 & $24.6(22.9)$ \\
\hline & $17.47-42.59$ & $16.25-161.90$ & 6 & $60.2(58.4)$ \\
\hline${ }^{131 \mathrm{~m}} \mathrm{Xe}$ & $17.47-42.59$ & $96.99-161.90$ & 7 & $78.0(74.9)$ \\
\hline${ }^{133 m} \mathrm{Xe}$ & $17.47-42.59$ & $170.07-239.03$ & 8 & $69.6(65.3)$ \\
\hline${ }^{135} \mathrm{Xe}$ & $226.58-275.47$ & 0-908.0 & 9 & $72.9(63.8)$ \\
\hline
\end{tabular}

Data in brackets is simulated with original PW5.

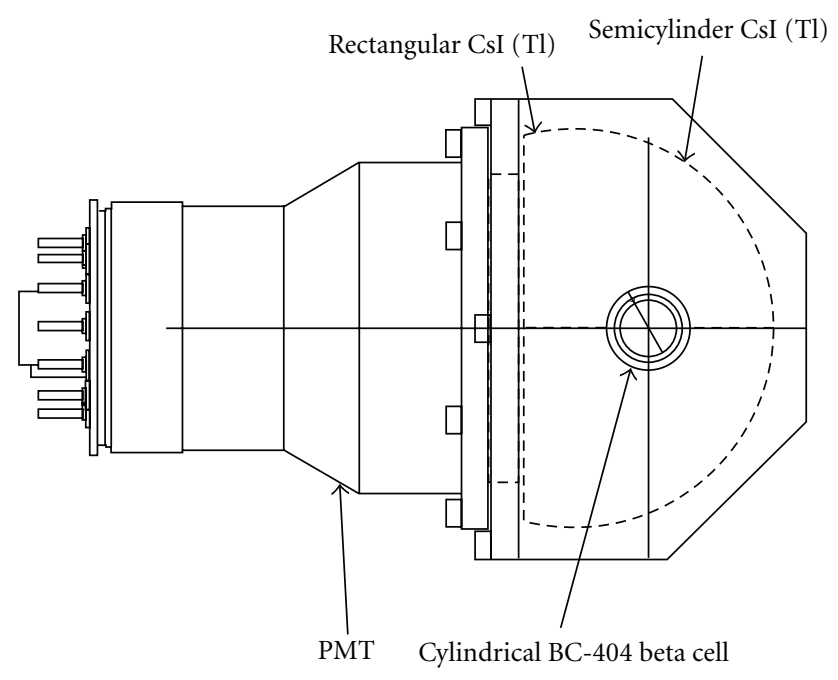

FIGURE 2: The schematic diagram of proposed detector geometry modeling by Geant4 simulations.

detector's central axis directly into PMT, reducing the average number of reflections per photon. Simulations were performed to optimize the FWHM of the $129.4 \mathrm{keV}$ CE of ${ }^{131 \mathrm{~m} X e}$ by varying the depth of the reflector and the displacement of the beta counting cell. Unfortunately, no significant improvement was observed. Although attempts were made to minimize the shadowing effect of the beta counting cell, the large size of the counting cell still limits the reduction that can be achieved to correct its light path interruption effect.

The maximum energy resolution observed so far was a $32.7 \%$ FWHM for the ${ }^{131 \mathrm{~m}}$ Xe CE peak by the dual PMT system. However, in order to approach this maximum energy resolution and retain the advantages (simpler electronics and algorithms and smaller size) of the single-channel system, a new design was proposed in this study. As shown in Figure 2, the BC404 has been changed to a cylindrical pipe, which has an outer diameter of $1.57 \mathrm{~cm}$, a wall thickness of $0.155 \mathrm{~cm}$ and length of $7.35 \mathrm{~cm}$, to minimize its light shadowing and scattering effect as a result of being an installed counting cell. The newly designed CsI( $\mathrm{Tl})$ crystal consists of a rectangular component $(10.16 \times 10.16 \times 1.27 \mathrm{~cm})$ and a semicylindrical scintillation component $(5.08 \mathrm{~cm}$ radius), which is used as a light reflector to increase light gathering. These scintillator geometries are commercially available. The readout electronics of the simulated system described in this study are based on the PW5 product package that is currently being beta-tested at the CTBT Canadian xenon radioisotope laboratory in Hennig et al. [10]. For each coincidence event, the ratio of the number of photons captured by the PMT to the number of photons produced from BC404 by ${ }^{131 \mathrm{~m}} \mathrm{Xe}$ $129.4 \mathrm{keV} \mathrm{CE}$ is calculated. The performance evaluation for each detector design is based on the histogram analysis of the ratios. The histogram plots in Figure 3 illustrate the best- and worst-case scenarios. The histogram of the worstcase scenario, as shown in Figure 3(a), has two broad peaks which are distributed around 0.17 and 0.20 , respectively, and the maximum of photon collection rate is about $40 \%$. With this detector design, the FWHM is calculated as $39.1 \%$, which suggests the design needs further improvement. The best-case scenario corresponds to the proposed detector design in this study. The histogram is a single peak and narrowly distributed around a mean value of 0.21 , as shown in Figure 3(b), and the maximum of the photon collection rate from BC404 can reach as high as 100\%. The FWHM of ${ }^{131 \mathrm{~m}} \mathrm{Xe} 129.4 \mathrm{keV} \mathrm{CE}$ is calculated as $33.1 \%$ in this best-case scenario. The beta-gamma coincidence detection efficiencies of the four xenon radioisotopes of interest, as listed in Table 1, are also calculated with the simulation results both at the proposed detector design in this study and original PW5 detector geometries using the same BC404 resolution scales. The simulated results in Table 1 indicate that the radioxenon coincidence counting efficiencies by the new detector design are about $5 \%$ to $14 \%$ better than those achieved with original PW5 system.

\section{Conclusions}

The MC simulation study demonstrated that the newly proposed single-channel phoswich well detector design has improved light collection by using a cylindrical pipe shaped beta cell in the $\mathrm{CsI}(\mathrm{Tl})$ crystal and a semicylindrical light reflector. Compared with PW5 detector, the final optimized 


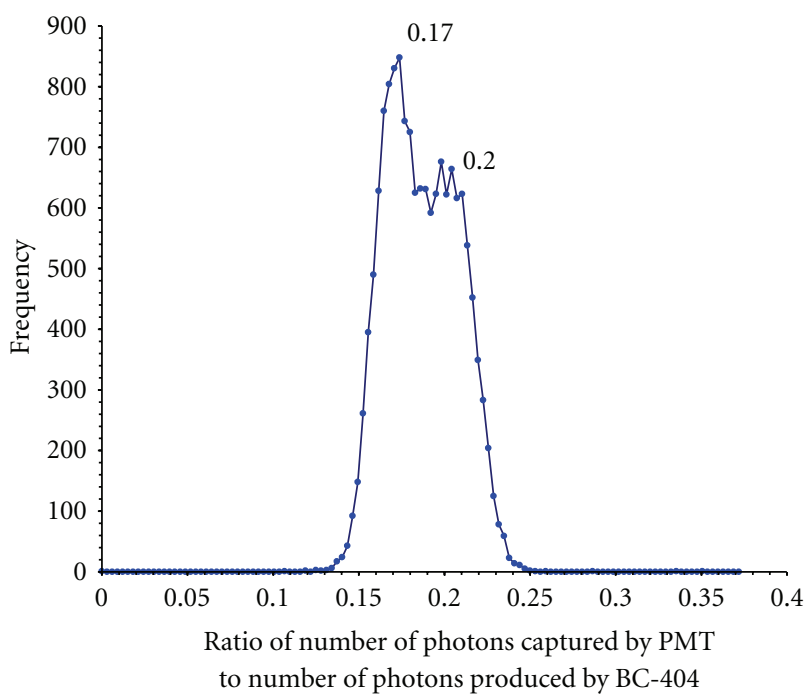

(a)

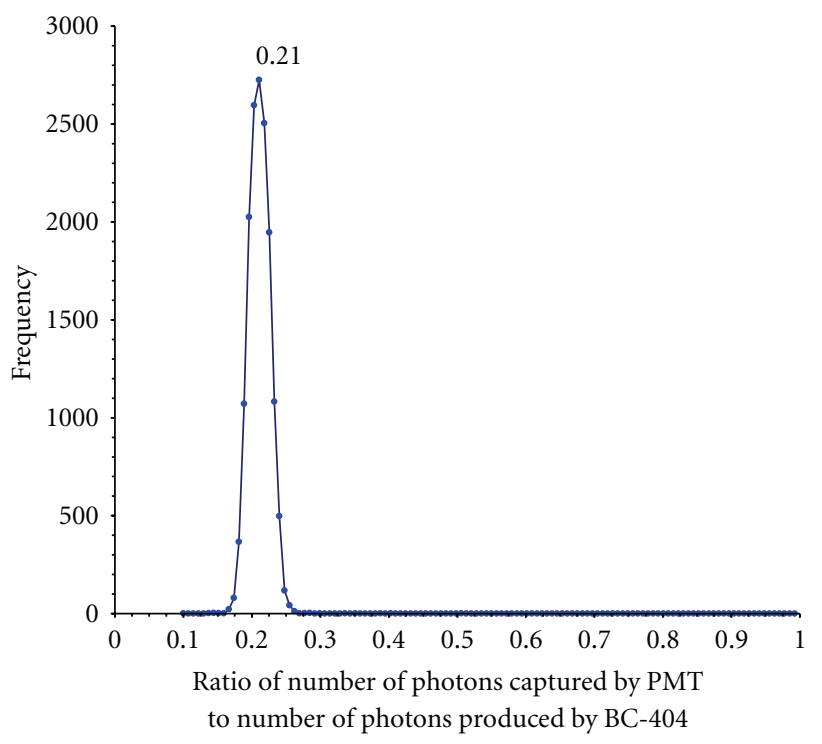

(b)

FIGURE 3: The BC404 photon counting histogram analysis for optimizing detector design parameters, (a) the worst-case scenario and (b) the best-case scenario.

detector geometry showed 15\% improvement in the energy resolution of the ${ }^{131 \mathrm{~m}} \mathrm{Xe} 129.4 \mathrm{keV}$ CE peak. Based on the simulations scaled to the empirical FWHM the ${ }^{131 \mathrm{~m}} \mathrm{Xe}$ $129.4 \mathrm{keV}$ CE peak, the calculated beta-gamma coincidence efficiencies of xenon radioisotopes are significantly better than those obtained with a PW5 detector.

\section{References}

[1] P. R. J. Saey and L. E. De Geer, "Notes on radioxenon measurements for CTBT verification purposes," Applied Radiation and Isotopes, vol. 63, no. 5-6, pp. 765-773, 2005.

[2] P. L. Reeder, T. W. Bowyer, and R. W. Perkins, "Betagamma counting system for Xe fission products," Journal of
Radioanalytical and Nuclear Chemistry, vol. 235, no. 1-2, pp. 89-94, 1998.

[3] A. Ringbom, T. Larson, A. Axelsson, K. Elmgren, and C. Johansson, "SAUNA - a system for automatic sampling, processing, and analysis of radioactive xenon," Nuclear Instruments and Methods in Physics Research Section A, vol. 508, no. 3, pp. 542-553, 2003.

[4] Y. S. Popov, Y. V. Dubasov et al., "Russian Federation, Automatic Portable Radiometer of Isotopes Xe ARIX-02 in Atmospheric Air and Subsoil Gas for On-Site Inspection,” Tech. Rep. CTBT/OSI/WS-6/PR/15, 2000.

[5] W. Hennig, H. Tan, W. K. Warburton, and J. I. Mcintyre, "Single-channel BetaGamma coincidence detection of radioactive xenon using digital pulse shape analysis of phoswich detector signals," IEEE Transactions on Nuclear Science, vol. 53, no. 2, pp. 620-624, 2006.

[6] W. Zhang, P. Mekarski, and K. Ungar, "Beta-gamma coincidence counting efficiency and energy resolution optimization by Geant4 Monte Carlo simulations for a phoswich well detector," Applied Radiation and Isotopes, 2010.

[7] S. Agostinelli, J. Allison, K. Amako et al., "GEANT4a simulation toolkit," Nuclear Instruments and Methods in Physics Research. Section A, vol. 506, no. 3, pp. 250-303, 2003.

[8] V. Innocente, L. Moneta, and A. Pfeiffer, "Review of the Abstract Interfaces for Data Analysis (AIDA) from a developer's perspective," CERN Version1.0, 2003.

[9] P. Mekarski, W. Zhang, K. Ungar, M. Bean, and E. Korpach, "Monte Carlo simulation of a PhosWatch detector using Geant4 for xenon isotope beta-gamma coincidence spectrum profile and detection efficiency calculations," Applied Radiation and Isotopes, vol. 67, no. 10, pp. 1957-1963, 2009.

[10] W. Hennig, H. Tan, W. K. Warburton et al., "Development of a COTS radioxenon detector system using Phoswich detectors and pulse shape analysis," Monitoring Research Review: Ground-Based Nuclear Explosion, 2008. 

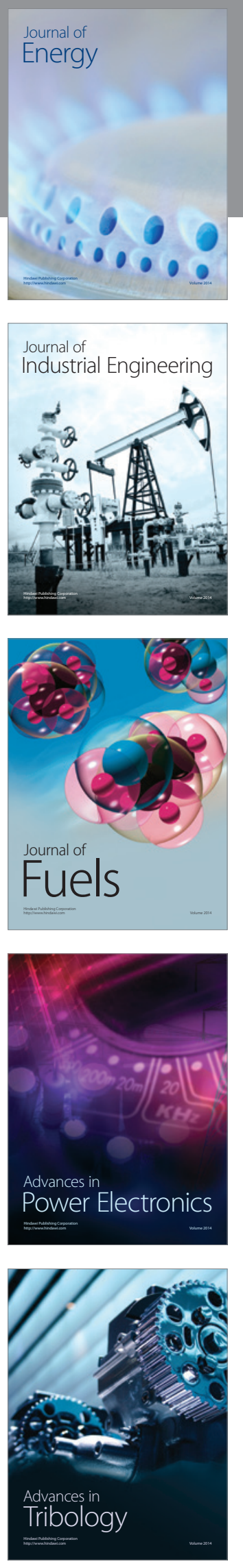
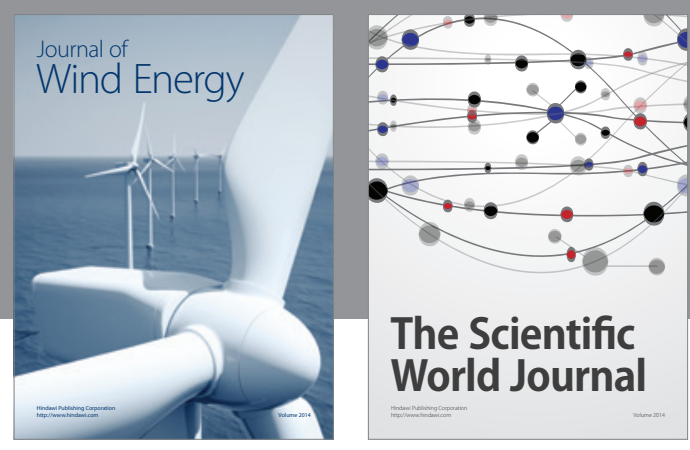

The Scientific World Journal

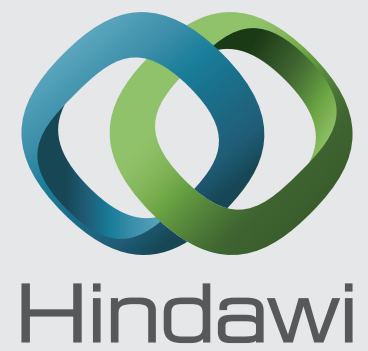

Submit your manuscripts at http://www.hindawi.com
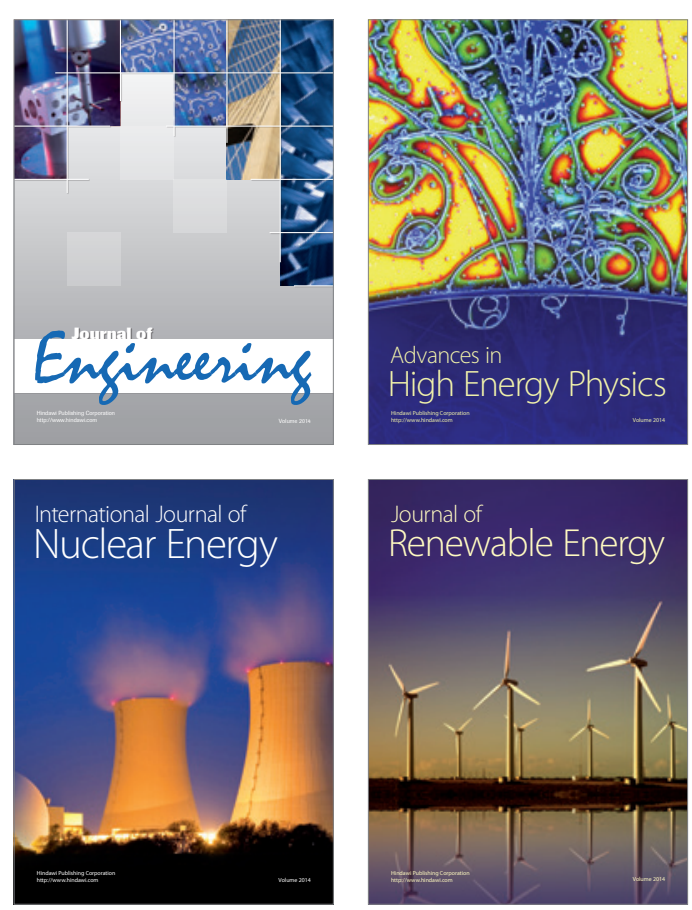

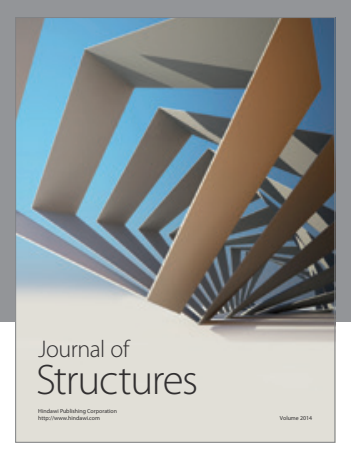

Rotating
Mechinery
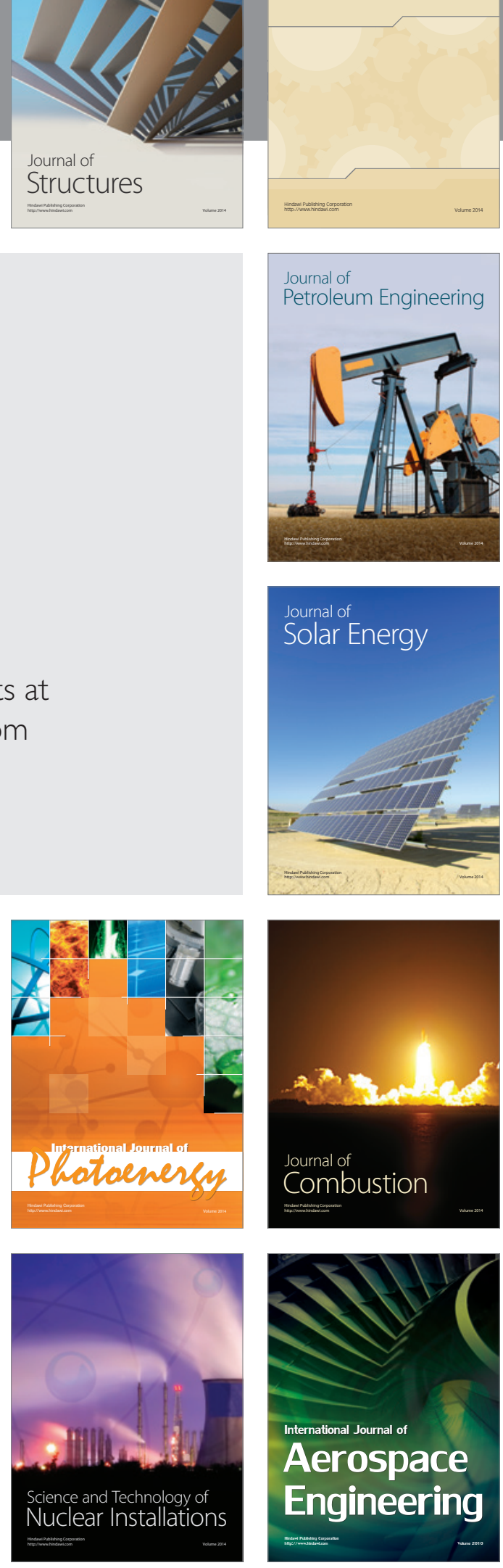